\title{
Sharp Stolarsky mean bounds for the complete elliptic integral of the second kind
}

\author{
Zhen-Hang Yang ${ }^{a, b}$, Yu-Ming Chu ${ }^{a, *}$, Xiao-Hui Zhang ${ }^{c}$ \\ a School of Mathematics and Computation Sciences, Hunan City University, Yiyang 413000, China. \\ ${ }^{b}$ Customer Service Center, State Grid Zhejiang Electric Power Research Institute, Hangzhou 310009, China. \\ ${ }^{c}$ Department of Mathematics, Zhejiang Sci-Tech University, Hangzhou 310018, China.
}

Communicated by Sh. Wu

\begin{abstract}
In the article, we prove that the double inequality

$$
25 / 16<\mathcal{E}(\mathrm{r}) / S_{5 / 2,2}\left(1, \mathrm{r}^{\prime}\right)<\pi / 2
$$

holds for all $r \in(0,1)$ with the best possible constants $25 / 16$ and $\pi / 2$, where $r^{\prime}=\left(1-r^{2}\right)^{1 / 2}, \mathcal{E}(r)=\int_{0}^{\pi / 2} \sqrt{1-r^{2} \sin ^{2}(t)} d t$, is the complete elliptic integral of the second kind and $S_{p, q}(a, b)=\left[q\left(a^{p}-b^{p}\right) /\left(p\left(a^{q}-b^{q}\right)\right)\right]^{1 /(p-q)}$, is the Stolarsky mean of a and b. (C) 2017 All rights reserved.
\end{abstract}

Keywords: Gaussian hypergeometric function, complete elliptic integral, Stolarsky mean. 2010 MSC: 33E05, 26D15, 26E60.

\section{Introduction}

For $r \in(0,1)$, the complete elliptic integrals $\mathcal{K}(r)$ and $\mathcal{E}(r)$ [1] of the first and second kinds are respectively given by

$$
\begin{aligned}
& \mathcal{K}(r)=\int_{0}^{\pi / 2} \frac{d t}{\sqrt{1-r^{2} \sin ^{2}(t)}}=\frac{\pi}{2} F\left(\frac{1}{2}, \frac{1}{2} ; 1 ; r^{2}\right)=\frac{\pi}{2} \sum_{n=0}^{\infty} \frac{\left(\frac{1}{2}\right)_{n}^{2}}{(n !)^{2}} r^{2 n}, \\
& \mathcal{E}(r)=\int_{0}^{\pi / 2} \sqrt{1-r^{2} \sin ^{2}(t)} d t=\frac{\pi}{2} F\left(-\frac{1}{2}, \frac{1}{2} ; 1 ; r^{2}\right)=\frac{\pi}{2} \sum_{n=0}^{\infty} \frac{\left(-\frac{1}{2}\right)_{n}\left(\frac{1}{2}\right)_{n}}{(n !)^{2}} r^{2 n},
\end{aligned}
$$

\footnotetext{
*Corresponding author

Email addresses: yzhkm@163.com (Zhen-Hang Yang), chuyuming2005@126.com (Yu-Ming Chu), xiaohui.zhang@zstu.edu.cn (Xiao-Hui Zhang)

doi:10.22436/jnsa.010.03.06
}

Received 2016-08-01 
where

$$
\mathrm{F}(\mathrm{a}, \mathrm{b} ; \mathrm{c} ; \mathrm{x})=\sum_{n=0}^{\infty} \frac{(\mathrm{a})_{n}(\mathrm{~b})_{\mathrm{n}}}{(\mathrm{c})_{\mathrm{n}}} \frac{x^{\mathrm{n}}}{\mathrm{n} !}, \quad(-1<x<1)
$$

is the Gaussian hypergeometric function, $(a)_{n}=\Gamma(a+n) / \Gamma(a)$ and $\Gamma(x)=\int_{0}^{\infty} t^{x-1} e^{-t} d t(x>0)$ is the gamma function. We clearly see that $\mathcal{K}(r)$ and $\mathcal{E}(r)$ satisfy the identities

$$
\mathcal{K}\left(0^{+}\right)=\mathcal{E}\left(0^{+}\right)=\frac{\pi}{2}, \quad \mathcal{K}\left(1^{-}\right)=\infty, \quad \mathcal{E}\left(1^{-}\right)=1 .
$$

It is well-known that the double inequality

$$
\frac{\pi}{2} A_{3 / 2}\left(1, r^{\prime}\right)<\mathcal{E}(r)<\frac{\pi}{2} A_{2}\left(1, r^{\prime}\right),
$$

holds for all $r \in(0,1)$ (see $[12,19.9 .4])$. Here and in what follows $r^{\prime}=\left(1-r^{2}\right)^{1 / 2}, A_{p}(a, b)=\left[\left(a^{p}+\right.\right.$ $\left.\left.b^{p}\right) / 2\right]^{1 / p}$. The first inequality of (1.2) is due to Qiu and Shen [13] and the second inequality of (1.2) is due to Barnard et al. [2].

In $[4,5]$, the authors proved that the inequalities

$$
\begin{aligned}
& \mathcal{E}(r)<\frac{\pi}{2} L_{1 / 4}\left(1, r^{\prime}\right), \\
& \mathcal{E}(r)<\frac{\pi}{32}\left[18 A\left(1, r^{\prime}\right)-5 G\left(1, r^{\prime}\right)+3 Q\left(1, r^{\prime}\right)\right],
\end{aligned}
$$

hold for all $r \in(0,1)$, where $A(a, b)=(a+b) / 2, G(a, b)=\sqrt{a b}, Q(a, b)=\sqrt{\left(a^{2}+b^{2}\right) / 2}$ and $L_{p}(a, b)=$ $\left(a^{p+1}+b^{p+1}\right) /\left(a^{p}+b^{p}\right)$ are the arithmetic, geometric, quadratic and $p$-th Lehmer means of $a$ and $b$, respectively. by

Let $p, q \in \mathbb{R}$ with $p \neq q$ and $p q \neq 0$, and $a, b>0$. Then the Stolarsky mean $S_{p, q}(a, b)[14]$ is defined

$$
S_{p, q}(a, b)=\left[\frac{q\left(a^{p}-b^{p}\right)}{p\left(a^{q}-b^{q}\right)}\right]^{1 /(p-q)}, \quad(a \neq b), \quad S_{p, q}(a, a)=a .
$$

Recently, the Stolarsky mean $S_{p, q}(a, b)$ has attracted the attention of many researchers. In particular, many remarkable inequalities involving the Stolarsky mean $S_{p, q}(a, b)$ can be found in the literature [6-11].

The main purpose of this paper is to present the best possible constants $\lambda$ and $\mu$ such that the double inequality

$$
\lambda<\mathcal{E}(\mathrm{r}) / \mathrm{S}_{5 / 2,2}\left(1, \mathrm{r}^{\prime}\right)<\mu,
$$

holds for all $r \in(0,1)$. Some complicated computations are carried out using Mathematica computer algebra system.

\section{Lemmas}

Lemma 2.1 ([3]). Let $A(t)=\sum_{k=0}^{\infty} a_{k} t^{k}$ and $B(t)=\sum_{k=0}^{\infty} b_{k} t^{k}$ be two real power series converging on $(-r, r)$ $(\mathrm{r}>0)$ with $\mathrm{b}_{\mathrm{k}}>0$ for all $\mathrm{k}$. If the non-constant sequence $\left\{\mathrm{a}_{\mathrm{k}} / \mathrm{b}_{\mathrm{k}}\right\}_{\mathrm{k}=0}^{\infty}$ is increasing (decreasing) for all $\mathrm{k}$, then the function $\mathrm{t} \mapsto \mathrm{A}(\mathrm{t}) / \mathrm{B}(\mathrm{t})$ is strictly increasing (decreasing) on $(0, \mathrm{r})$.

Lemma 2.2 ([5]). The inequality

$$
\mathrm{L}_{1 / 4}(\mathrm{a}, \mathrm{b})>\frac{18 \mathrm{~A}(\mathrm{a}, \mathrm{b})-5 \mathrm{G}(\mathrm{a}, \mathrm{b})+3 \mathrm{Q}(\mathrm{a}, \mathrm{b})}{16}
$$

holds for all $\mathrm{a}, \mathrm{b}>0$ with $\mathrm{a} \neq \mathrm{b}$. 
Lemma 2.3. Let $\mathrm{n}=1,2,3, \cdots$, and $\mathrm{u}_{\mathrm{n}}$ and $v_{\mathrm{n}}$ be defined by

$$
u_{n}=\frac{6\left(\frac{1}{2}\right)_{n-1}+2\left(\frac{3}{4}\right)_{n}}{5(n+2) !}, \quad v_{n}=\frac{\left(\frac{1}{2}\right)_{n-1}\left(\frac{1}{2}\right)_{n}}{2(n !)^{2}} .
$$

Then the non-constant sequence $\left\{v_{n} / u_{n}\right\}_{n=1}^{\infty}$ is increasing for all $n \geqslant 1$.

Proof. Let

$$
w_{n}=\frac{v_{n+1} u_{n}}{v_{n}}-u_{n+1} \text {. }
$$

Then it follows from (2.1) that

$$
w_{n}=\frac{6(3 n+1)\left(\frac{1}{2}\right)_{n}+\left(n^{2}-11 n-6\right)\left(\frac{3}{4}\right)_{n}}{10(n+1)^{2}(n+3) !} .
$$

Elaborated computations lead to

$$
\begin{aligned}
& w_{1}=w_{2}=0, \quad w_{3}=\frac{3}{81920}, \quad w_{4}=\frac{21}{512000}, \quad w_{5}=\frac{47}{1310720}, \\
& w_{6}=\frac{1881}{64225280}, \quad w_{7}=\frac{157531}{6710886400}, \quad w_{8}=\frac{42559}{2264924160}, \\
& w_{9}=\frac{507577}{33554432000}, \quad w_{10}=\frac{997177}{81201725440}, \quad w_{11}=\frac{20743573}{2061584302080} .
\end{aligned}
$$

Note that

$$
n^{2}-11 n-6>0
$$

for all $n \geqslant 12$.

Therefore, Lemma 2.3 follows easily from (2.2), (2.3), (2.4), (2.5), (2.6), (2.7).

Lemma 2.4. The inequality

$$
\mathrm{L}_{1 / 4}(1, x)>\mathrm{S}_{5 / 2,2}(1, x)
$$

holds for all $x \in(0,1)$.

Proof. It follows from (1.5) that

$$
\begin{aligned}
\mathrm{L}_{1 / 4}(1, x)-\mathrm{S}_{5 / 2,2}(1, x)= & \frac{1+x^{5 / 4}}{1+x^{1 / 4}}-\frac{16\left(1-x^{5 / 2}\right)^{2}}{25\left(1-x^{2}\right)^{2}} \\
= & \frac{\left(1-x^{1 / 4}\right)^{6}\left(1+x^{1 / 4}\right)\left(1+x^{5 / 4}\right)}{25\left(1-x^{2}\right)^{2}} \\
& \times\left(9 x^{2}+20 x^{7 / 4}+44 x^{3 / 2}+60 x^{5 / 4}+74 x+60 x^{3 / 4}+44 x^{1 / 2}+20 x^{1 / 4}+9\right)>0 .
\end{aligned}
$$

Lemma 2.5. Let $\mathrm{f}(\mathrm{x})$ and $\mathrm{g}(\mathrm{x})$ be defined by

$$
\begin{aligned}
& f(x)=S_{5 / 2,2}\left(1, x^{2}\right) \\
& g(x)=\frac{9}{8} A\left(1, x^{2}\right)-\frac{5}{16} G\left(1, x^{2}\right)+\frac{3}{16} Q\left(1, x^{2}\right) .
\end{aligned}
$$

Then there exists $x_{0} \in(0,1)$ such that $\mathrm{f}(\mathrm{x})>\mathrm{g}(\mathrm{x})$, for $\mathrm{x} \in\left(0, \mathrm{x}_{0}\right)$ and $\mathrm{f}(\mathrm{x})<\mathrm{g}(\mathrm{x})$, for $\mathrm{x} \in\left(\mathrm{x}_{0}, 1\right)$. 
Proof. Let $x \in(0,1), u=x+1 / x \in(2, \infty), v=u-2=x+1 / x-2 \in(0, \infty), h_{1}(x), h_{2}(x)$ and $h(x)$ be defined by

$$
\begin{aligned}
& h_{1}(x)=\frac{31 x^{8}+187 x^{7}+118 x^{6}+49 x^{5}+430 x^{4}+49 x^{3}+118 x^{2}+187 x+31}{\left(x^{2}+1\right)^{2}(x+1)^{2}} \\
& h_{2}(x)=\frac{75 \sqrt{2} \sqrt{1+x^{4}}}{2}, \\
& h(x)=3703 x^{6}+14124 x^{5}-16260 x^{4}-98560 x^{3}-23040 x^{2}+98304 x-32768 .
\end{aligned}
$$

Then elaborated computations give

$$
\begin{gathered}
f(x)-g(x)=\frac{16\left(1-x^{5}\right)^{2}}{25\left(1-x^{4}\right)^{2}}-\left[\frac{9\left(1+x^{2}\right)}{16}-\frac{5 x}{16}+\frac{3}{16} \sqrt{\frac{1+x^{4}}{2}}\right], \\
=\frac{h_{1}(x)-h_{2}(x)}{400}=\frac{h_{1}^{2}(x)-h_{2}^{2}(x)}{400\left[h_{1}(x)+h_{2}(x)\right]} \\
h_{1}(x)=\frac{31 u^{4}+187 u^{3}-6 u^{2}-512 u+256}{u^{2}(u+2)} x, \quad h_{2}(x)=75 x \sqrt{\frac{u^{2}-2}{2}}, \\
h_{1}^{2}(x)-h_{2}^{2}(x)=-\frac{x^{2}(u-2)^{2}}{2 u^{4}(u+2)^{2}} h(u), \\
h(u)=h(v+2)=3703 v^{6}+58560 v^{5}+347160 v^{4}+928800 v^{3}+1014000 v^{2}+144000 v-288000 .
\end{gathered}
$$

From (2.10) we clearly see that there exists $v_{0} \in(0, \infty)$ such that $h(v+2)<0$ for $v \in\left(0, v_{0}\right)$ and $\mathrm{h}(v+2)>0$ for $v \in\left(v_{0}, \infty\right)$. Then (2.8), (2.9), (2.10) lead to Lemma 2.5. Numerical computations show that $v_{0}=0.3994 \cdots$ and $x_{0}=\left[v_{0}+2-\sqrt{v_{0}\left(v_{0}+4\right)}\right] / 2=0.5368 \cdots$.

\section{Main results}

Theorem 3.1. The double inequality

$$
\lambda S_{5 / 2,2}\left(1, r^{\prime}\right)<\mathcal{E}(r)<\mu S_{5 / 2,2}\left(1, r^{\prime}\right),
$$

holds for all $r \in(0,1)$, if and only if $\lambda \leqslant 25 / 16=1.5625$ and $\mu \geqslant \pi / 2=1.5707 \cdots$.

Proof. Let $r \in(0,1), u_{n}$ and $v_{n}$ be defined by (2.1) and

$$
F(r)=\frac{1-\frac{2}{\pi} \varepsilon(r)}{1-S_{5 / 2,2}\left(1, r^{\prime}\right)}
$$

Then it follows from (1.1), (1.5), (2.1), (3.1) and $(a)_{n}=a(a+1)_{n-1}$ that

$$
\begin{gathered}
\mathrm{u}_{1}=v_{1}=\frac{1}{4}, \\
\mathrm{~F}\left(1^{-}\right)=\frac{25(\pi-2)}{9 \pi}, \\
1-\frac{2}{\pi} \varepsilon(r)=-\sum_{n=1}^{\infty} \frac{\left(-\frac{1}{2}\right)_{n}\left(\frac{1}{2}\right)_{n}}{(n !)^{2}} r^{2 n}=\sum_{n=1}^{\infty} v_{n} r^{2 n},
\end{gathered}
$$




$$
\begin{aligned}
1-S_{5 / 2,2}\left(1, r^{\prime}\right) & =1-\frac{16\left[\left(1-r^{2}\right)^{5 / 2}-2\left(1-r^{2}\right)^{5 / 4}+1\right]}{25 r^{4}} \\
& =1-\frac{16}{25 r^{4}}\left[\sum_{n=0}^{\infty} \frac{\left(-\frac{5}{2}\right)_{n}}{n !} r^{2 n}-2 \sum_{n=0}^{\infty} \frac{\left(-\frac{5}{4}\right)_{n}}{n !} r^{2 n}+1\right] \\
& =\frac{16}{25} \sum_{n=1}^{\infty} \frac{2\left(-\frac{5}{4}\right)_{n+2}-\left(-\frac{5}{2}\right)_{n+2}}{(n+2) !} r^{2 n}=\sum_{n=1}^{\infty} u_{n} r^{2 n}
\end{aligned}
$$

From Lemmas 2.1 and $2.3,(3.1),(3.2),(3.4)$ and (3.5) we know that $F(r)$ is strictly increasing on $(0,1)$ and

$$
\mathrm{F}\left(0^{+}\right)=\frac{v_{1}}{\mathrm{u}_{1}}=1 \text {. }
$$

Equations (3.1), (3.3) and (3.6) together with the monotonicity of $F(r)$ on the interval $(0,1)$ lead to the conclusion that

$$
-\frac{8 \pi-25}{9}+\frac{25(\pi-2)}{18} S_{5 / 2,2}\left(1, r^{\prime}\right)<\mathcal{E}(r)<\frac{\pi}{2} S_{5 / 2,2}\left(1, r^{\prime}\right)
$$

It follows from (1.5) that

$$
\begin{aligned}
-\frac{8 \pi-25}{9}+\frac{25(\pi-2)}{18} S_{5 / 2,2}\left(1, \mathrm{r}^{\prime}\right)-\frac{25}{16} S_{5 / 2,2}\left(1, \mathrm{r}^{\prime}\right) & =\frac{25(8 \pi-25)}{144}\left(S_{5 / 2,2}\left(1, \mathrm{r}^{\prime}\right)-\frac{16}{25}\right) \\
& >\frac{25(8 \pi-25)}{144}\left(S_{5 / 2,2}\left(1,0^{+}\right)-\frac{16}{25}\right)=0,
\end{aligned}
$$

for $r \in(0,1)$ and

$$
\lim _{r \rightarrow 0^{+}} \frac{\varepsilon(r)}{S_{5 / 2,2}\left(1, r^{\prime}\right)}=\frac{\pi}{2}, \quad \lim _{r \rightarrow 1^{-}} \frac{\varepsilon(r)}{S_{5 / 2,2}\left(1, r^{\prime}\right)}=\frac{25}{16} .
$$

Therefore, Theorem 3.1 follows from (3.7), (3.8), (3.9).

From Theorem 3.1 we get Corollary 3.2 immediately.

Corollary 3.2. The double inequality

$$
3.125<\frac{2 \mathcal{E}(\mathrm{r})}{\mathrm{S}_{5 / 2,2}\left(1, \mathrm{r}^{\prime}\right)}<\pi,
$$

holds for all $r \in(0,1)$.

Corollary 3.3. The inequality

$$
\mathcal{E}(\mathrm{r})>\frac{\pi}{2} S_{5 / 2,2}\left(1, \mathrm{r}^{\prime}\right)-\left(\frac{8 \pi}{25}-1\right)
$$

holds for all $\mathrm{r} \in(0,1)$.

Proof. Let $r \in(0,1), F(r)$ be defined by (3.1) and $H(r)$ be defined by

$$
H(r)=S_{5 / 2,2}\left(1, r^{\prime}\right)-\frac{2}{\pi} \varepsilon(r) .
$$

Then

$$
\mathrm{H}(\mathrm{r})=[\mathrm{F}(\mathrm{r})-1]\left[1-\mathrm{S}_{5 / 2,2}\left(1, \mathrm{r}^{\prime}\right)\right] .
$$

We clearly see that the function $r \mapsto 1-S_{5 / 2,2}\left(1, r^{\prime}\right)$ is strictly increasing from $(0,1)$ onto $(0,9 / 25)$. From the proof of Theorem 3.1 we know that $F(r)-1$ is strictly increasing from $(0,1)$ onto $(0,2(8 \pi-25) /(9 \pi))$. Then (3.11) leads to the conclusion that $H(r)$ is strictly increasing from $(0,1)$ onto $(0,16 / 25-2 / \pi)$ and

$$
\mathrm{H}(\mathrm{r})<\frac{16}{25}-\frac{2}{\pi}
$$

for all $r \in(0,1)$. Therefore, Corollary 3.3 follows from (3.10) and (3.12). 
Remark 3.4. Extensive numerical computations show that in all cases the upper bound for $\mathcal{E}(\mathrm{r})$ in Theorem 3.1 is sharper than the corresponding upper bound derived from (1.2), but the lower bound for $\mathcal{E}(\mathrm{r})$ derived from (1.2) is better than that obtained with the use of Theorem 3.1.

Remark 3.5. For all $r \in(0,1)$, from Lemmas 2.2, 2.4 and 2.5, we clearly see that the upper bounds $\pi \mathrm{S}_{5 / 2,2}\left(1, \mathrm{r}^{\prime}\right) / 2$ and $\pi\left[18 \mathrm{~A}\left(1, \mathrm{r}^{\prime}\right)-5 \mathrm{G}\left(1, \mathrm{r}^{\prime}\right)+3 \mathrm{Q}\left(1, \mathrm{r}^{\prime}\right)\right] / 32$ for $\mathcal{E}(\mathrm{r})$ given in Theorem 3.1 and $(1.4)$ are better than the upper bound $\pi \mathrm{L}_{1 / 4}\left(1, \mathrm{r}^{\prime}\right) / 2$ given in (1.3) and there exists $r_{0} \in(0,1)$ such that the upper bound $\pi S_{5 / 2,2}\left(1, r^{\prime}\right) / 2$ for $\mathcal{E}(r)$ given in Theorem 3.1 is better than the upper bound $\pi\left[18 A\left(1, r^{\prime}\right)-5 G\left(1, r^{\prime}\right)+\right.$ $\left.3 \mathrm{Q}\left(1, \mathrm{r}^{\prime}\right)\right] / 32$ given in (1.4) for $\mathrm{r} \in\left(0, \mathrm{r}_{0}\right)$.

Remark 3.6. Let $\mathrm{r} \in(0,1), \triangle_{1}(\mathrm{r})=\mathrm{L}_{1 / 4}\left(1, \mathrm{r}^{\prime}\right)-2 \mathcal{E}(\mathrm{r}) / \pi, \triangle_{2}(\mathrm{r})=\left[18 \mathrm{~A}\left(1, \mathrm{r}^{\prime}\right)-5 \mathrm{G}\left(1, \mathrm{r}^{\prime}\right)+3 \mathrm{Q}\left(1, \mathrm{r}^{\prime}\right)\right] / 16-$ $2 \mathcal{E}(\mathrm{r}) / \pi$ and $\triangle_{3}(\mathrm{r})=S_{5 / 2,2}\left(1, \mathrm{r}^{\prime}\right)-2 \mathcal{E}(\mathrm{r}) / \pi$. Then making use of power series formulas and Corollary 3.3 we get

$$
\begin{aligned}
& \triangle_{1}(r)=\frac{1}{2^{12}} r^{8}+o\left(r^{8}\right), \triangle_{2}(r)=\frac{7}{2^{20}} r^{12}+o\left(r^{12}\right), \triangle_{3}(r)=\frac{3}{5 \times 2^{14}} r^{8}+o\left(r^{8}\right), \\
& \sup _{r \in(0,1)} \triangle_{1}(r) \geqslant \triangle_{1}\left(1^{-}\right)=1-\frac{2}{\pi}=0.3633802276 \cdots, \\
& \sup _{r \in(0,1)} \triangle_{2}(r) \geqslant \triangle_{2}\left(1^{-}\right)=\frac{18+3 \sqrt{2}}{32}-\frac{2}{\pi}=0.0584627491 \cdots, \\
& \sup _{r \in(0,1)} \triangle_{3}(r) \leqslant \frac{16}{25}-\frac{2}{\pi}=0.0033802276 \cdots .
\end{aligned}
$$

Corollary 3.7. The double inequality

$$
\frac{\pi}{2} S_{5 / 2,2}\left(1, r^{\prime}\right)-\left(\frac{8 \pi}{25}-1\right) r^{8}<\mathcal{E}(r)<\frac{\pi}{2} S_{5 / 2,2}\left(1, r^{\prime}\right)-\frac{3 \pi}{5} \times 2^{-15} r^{8},
$$

holds for all $\mathrm{r} \in(0,1)$.

Proof. Let $u_{n}, v_{n}$ and $w_{n}$ be respectively defined by (2.1) and (2.2) and $\sigma_{n}$ be defined by

$$
\sigma_{n}=v_{n}-u_{n}
$$

Then it follows from (2.1), (2.2), (2.3), (2.4), (2.5), (2.6), (2.7) and (3.13) that

$$
\begin{gathered}
\sigma_{1}=\sigma_{2}=\sigma_{3}=0, \quad \sigma_{4}=\frac{3}{5} \times 2^{-14}, \\
\sigma_{n+1}-\frac{v_{n+1}}{v_{n}} \sigma_{n}=w_{n}>0,
\end{gathered}
$$

for all $n \geqslant 3$.

Inequality (3.15) leads to the conclusion that

$$
\sigma_{\mathrm{n}}>0,
$$

for all $n \geqslant 4$.

From (3.4), (3.5), (3.13), (3.14) and (3.16) we get

$$
\frac{S_{5 / 2,2}\left(1, r^{\prime}\right)-\frac{2}{\pi} \mathcal{E}(r)}{r^{8}}=\frac{3}{5} \times 2^{-14}+\sum_{n=5}^{\infty} \sigma_{n} r^{2 n-8}
$$


Equation (3.17) leads to

$$
\frac{3}{5} \times 2^{-14}<\frac{S_{5 / 2,2}\left(1, r^{\prime}\right)-\frac{2}{\pi} \varepsilon(r)}{r^{8}}<\lim _{r \rightarrow 1^{-}} \frac{S_{5 / 2,2}\left(1, r^{\prime}\right)-\frac{2}{\pi} \varepsilon(r)}{r^{8}}=\frac{16}{25}-\frac{2}{\pi^{\prime}}
$$

for $r \in(0,1)$.

Therefore, Corollary 3.7 follows easily from (3.18) .

Corollary 3.8. The inequality

$$
\mathcal{E}(r)<\frac{875 \pi}{1744} S_{5 / 2,2}\left(1, r^{\prime}\right)-\frac{3 \pi}{1744}\left(1-\frac{1}{4} r^{2}-\frac{3}{64} r^{4}-\frac{5}{256} r^{6}\right)
$$

holds for all $r \in(0,1)$.

Proof. Let $u_{n}, v_{n}$ and $\sigma_{n}$ be respectively defined by (2.1) and (3.13). Then it follows from Lemma 2.3, (2.1), (3.3), (3.4) and (3.13), (3.14), (3.15) that

$$
\sigma_{n}>\frac{\sigma_{n-1}}{v_{n-1}} v_{n}>\frac{\sigma_{4}}{v_{4}} v_{n}=\frac{\frac{3}{5} \times 2^{-14}}{175 \times 2^{-14}} v_{n}=\frac{3}{875} v_{n}
$$

for $n \geqslant 5$ and

$$
\begin{aligned}
S_{5 / 2,2}\left(1, r^{\prime}\right)-\frac{2}{\pi} \mathcal{E}(r) & =\sum_{n=4}^{\infty} \sigma_{n} r^{2 n}>\frac{3}{875} \sum_{n=4}^{\infty} v_{n} r^{2 n}=\frac{3}{875}\left(\sum_{n=1}^{\infty} v_{n} r^{2 n}-\sum_{n=1}^{3} v_{n} r^{2 n}\right) \\
& =\frac{3}{875}\left[1-\frac{2}{\pi} \mathcal{E}(r)-\left(\frac{1}{4} r^{2}+\frac{3}{64} r^{4}+\frac{5}{256} r^{6}\right)\right],
\end{aligned}
$$

for $r \in(0,1)$.

Therefore, Corollary 3.8 follows easily from (3.19).

\section{Acknowledgment}

The research was supported by the Natural Science Foundation of China under Grants 61673169, 61374086, 11371125, 11601485 and 11401191, and the Tianyuan Special Funds of the Natural Science Foundation of China under Grant 11626101.

\section{References}

[1] M. Abramowitz, I. A. Stegun, Handbook of mathematical functions with formulas, graphs, and mathematical tables, National Bureau of Standards Applied Mathematics Series, For sale by the Superintendent of Documents, U.S. Government Printing Office, Washington, D.C., (1964). 1

[2] R. W. Barnard, K. Pearce, K. C. Richards, A monotonicity property involving ${ }_{3} \mathrm{~F}_{2}$ and comparisons of the classical approximations of elliptical arc length, SIAM J. Math. Anal., 32 (2000), 403-419. 1

[3] M. Biernacki, J. Krzyż, On the monotonity of certain functionals in the theory of analytic functions, Ann. Univ. Mariae Curie-Skłodowska. Sect. A., 9 (1955), 135-147. 2.1

[4] Y.-M. Chu, M- K. Wang, Optimal Lehmer mean bounds for the Toader mean, Results Math., 61 (2012), 223-229. 1

[5] Y.-M. Chu, M.-K. Wang, S.-L. Qiu, Y.-P. Jiang, Bounds for complete elliptic integrals of the second kind with applications, Comput. Math. Appl., 63 (2012), 1177-1184. 1, 2.2

[6] P. Czinder, Z. Páles, Minkowski-type inequalities for two variable Stolarsky means, Acta Sci. Math. (Szeged), 69 (2003), 27-47. 1

[7] P. Czinder, Z. Páles, An extension of the Hermite-Hadamard inequality and an application for Gini and Stolarsky means, JIPAM. J. Inequal. Pure Appl. Math., 5 (2004), 8 pages.

[8] P. Czinder, Z. Páles, Some comparison inequalities for Gini and Stolarsky means, Math. Inequal. Appl., 9 (2006), 607616.

[9] L. Losonczi, Ratio of Stolarsky means: monotonicity and comparison, Publ. Math. Debrecen, 75 (2009), $221-238$. 
[10] E. Neuman, Z. Páles, On comparison of Stolarsky and Gini means, J. Math. Anal. Appl., 278 (2003), $274-284$.

[11] E. Neuman, J. Sándor, Inequalities involving Stolarsky and Gini means, Math. Pannon, 14 (2003), 29-44. 1

[12] F. W. J. Olver, D. W. Lozier, R. F. Boisvert, C. W. Clark, (Ed.) NIST handbook of mathematical functions, With 1 CD-ROM (Windows, Macintosh and UNIX). U.S. Department of Commerce, National Institute of Standards and Technology, Washington, DC; Cambridge University Press, Cambridge, (2010). 1

[13] S.-L. Qiu, J.-M. Shen, On two problems concerning means, (Chinese) J. Hangzhou Inst. Electron. Eng., 17 (1997), 1-7. 1

[14] K. B. Stolarsky, Generalizations of the logarithmic mean, Math. Mag., 48 (1975), 87-92. 1 